\title{
Placental gene-expression profiles of intrahepatic cholestasis of pregnancy reveal involvement of multiple molecular pathways in blood vessel formation and inflammation
}

QiaoLing Du ${ }^{1 \dagger}$, YouDong Pan ${ }^{2 \dagger}$, YouHua Zhang ${ }^{2 \dagger}$, Hailong Zhang ${ }^{2}$, YaJuan Zheng ${ }^{2}$, Ling Lu ${ }^{2}$, JunLei Wang ${ }^{2}$, Tao Duan $^{1 *}$ and JianFeng Chen ${ }^{2^{*}}$

\begin{abstract}
Background: Intrahepatic cholestasis of pregnancy (ICP) is a pregnancy-associated liver disease with potentially deleterious consequences for the fetus, particularly when maternal serum bile-acid concentration $>40 \mu \mathrm{M}$. However, the etiology and pathogenesis of ICP remain elusive. To reveal the underlying molecular mechanisms for the association of maternal serum bile-acid level and fetal outcome in ICP patients, DNA microarray was applied to characterize the whole-genome expression profiles of placentas from healthy women and women diagnosed with ICP.

Methods: Thirty pregnant women recruited in this study were categorized evenly into three groups: healthy group; mild $I C P$, with serum bile-acid concentration ranging from 10-40 $\mu \mathrm{M}$; and severe ICP, with bile-acid concentration $>40 \mu \mathrm{M}$. Gene Ontology analysis in combination with construction of gene-interaction and gene co-expression networks were applied to identify the core regulatory genes associated with ICP pathogenesis, which were further validated by quantitative real-time PCR and histological staining.

Results: The core regulatory genes were mainly involved in immune response, VEGF signaling pathway and G-protein-coupled receptor signaling, implying essential roles of immune response, vasculogenesis and angiogenesis in ICP pathogenesis. This implication was supported by the observed aggregated immune-cell infiltration and deficient blood vessel formation in ICP placentas.

Conclusions: Our study provides a system-level insight into the placental gene-expression profiles of women with mild or severe ICP, and reveals multiple molecular pathways in immune response and blood vessel formation that might contribute to ICP pathogenesis.
\end{abstract}

Keywords: Microarray, Intrahepatic cholestasis of pregnancy, Placenta, Genome-wide, Immune response

\section{Background}

Intrahepatic cholestasis of pregnancy (ICP) is the most frequent liver disease arising in the second or third trimester of pregnancy. Clinically, ICP is characterized by maternal pruritus, deranged liver function, elevated serum level of bile acids, and spontaneous relief of signs and symptoms

\footnotetext{
* Correspondence: tduan@yahoo.com; jfchen@sibcb.ac.cn

${ }^{\dagger}$ Equal contributors

'Department of Obstetrics, Shanghai First Maternity and Infant Hospital, Tongji University School of Medicine, Shanghai 200040, China

${ }^{2}$ State Key Laboratory of Cell Biology, Institute of Biochemistry and Cell Biology, Shanghai Institutes for Biological Sciences, Chinese Academy of Sciences, Shanghai 200031, China
}

(c) 2014 Du et al.; licensee BioMed Central Ltd. This is an Open Access article distributed under the terms of the Creative Commons Attribution License (http://creativecommons.org/licenses/by/2.0), which permits unrestricted use, distribution, and reproduction in any medium, provided the original work is properly credited. The Creative Commons Public Domain Dedication waiver (http://creativecommons.org/publicdomain/zero/1.0/) applies to the data made available in this article unless otherwise stated. in the mother within $2-3$ weeks after delivery $[1,2]$. The prevalence of this disease has been found to be variable, ranging from $0.1-4.0 \%[1,3]$. In contrast to the benign effects on the mother, ICP has severe consequences for the fetus: it is associated with an increased risk of fetal preterm delivery (19-60\% of ICP cases), fetal distress (22$41 \%)$, and fetal loss (0.4-1.6\%) [4]. However, the etiology and pathogenesis of ICP remain elusive.

An indispensible criterion for ICP is elevation in the serum levels of bile acids, which is considered to be the most appropriate laboratory parameter for diagnosis of the condition [5-7]. During a healthy pregnancy, bile acids

\section{Biomed Central}


are synthesized by the fetus from the 12th week of gestation and exported into the maternal circulation across a steep transplacental gradient [8,9]. In cases of maternal cholestasis, however, the serum levels of bile acids are elevated in both maternal and fetal serum, resulting in a reversal in the transplacental gradient and consequently impairing the export of bile acids from the fetus into the maternal circulation [10,11]. Rates of fetal complication are positively correlated with the total level of bile acids in maternal serum of ICP patients: the probability of fetal complications increases by $1-2 \%$ for each additional $1 \mu \mathrm{M}$ of bile acids, whereas the probability of these events did not increase until bile-acid levels $>40 \mu \mathrm{M}$ [6]. Although the association of maternal serum bile-acid level and fetal outcome has been reported, the underlying mechanisms remain unknown.

The placenta connects the developing fetus to the uterine wall and allows nutrient uptake, waste elimination, and gas exchange between the fetal and maternal blood supplies. The placenta is believed to play a critical role in ICP pathogenesis [12]. Previous studies using human placental tissues or a rodent model of ICP have revealed several morphological abnormalities in ICP placentas, including focally thickened amniotic basement membranes, chorionic villi that are small for gestational age, crowding and congestion of the villi, and an increased number of syncytial knots $[12,13]$. In addition, increased apoptosis, oxidative stress, and hypoxia have been observed in rat ICP placentas [12]. Thus, in this study, to reveal the molecular mechanisms for the association of maternal serum bile-acid level and fetal outcome in ICP patients, we categorized the ICP patients into two groups (mild ICP, with serum bile-acid concentration ranging from 10-40 $\mu \mathrm{M}$; severe ICP, with bile-acid concentration $>40 \mu \mathrm{M}$ ) and used DNA microarray to characterize the whole-genome expression profiles of placentas. Our system-level analyses of placental gene-expression profiles of ICP, coupled with validation of predicted tissue phenotypes, reveal multiple molecular pathways in immune response and blood vessel formation that might contribute to ICP pathogenesis.

\section{Methods}

\section{Ethics statement}

This study was approved by the Research Committee for Human Subjects, Shanghai Tongji University School of Medicine. All participants provided their written informed consent to participate in this study.

\section{Patients and tissues}

Thirty pregnant women recruited in this study were categorized into three groups (ten pregnancies in each group) according to the serum bile acid level at gestational age of 32 weeks: healthy group with serum bile acid concentration $<10 \mu \mathrm{M}$; mild ICP, with serum bile acid concentration ranging from $10-40 \mu \mathrm{M}$; and severe ICP, with bile acid concentration $>40 \mu \mathrm{M}$ (Figure 1A). ICP was diagnosed with the following symptoms: 1) presence of classical pruritus with no rash and quick disappearance after delivery; 2) raised maternal serum bile-acid concentration $(>10 \mu \mathrm{M}) ; 3)$ absence of itching skin disease; and 4) absence of any other causes of liver dysfunction, including preeclampsia; hemolysis, elevated liver enzymes and low platelets (HELLP) syndrome; acute fatty liver of pregnancy; primary biliary cirrhosis; viral hepatitis; and any ultrasound abnormality. All of the ICP patients were not treated with UDCA under their consents. Exclusion criteria for recruitment of healthy pregnant women were similar to that for ICP cases.

To avoid artefact from effects of labor, only placental samples obtained from women who had not undergone labor were selected. All samples were collected immediately after Caesarean sections, cut near the center zone of maternal surface villous lobule after deciduas and amnionic membranes were removed, and then rinsed with saline to remove maternal blood.

\section{Total serum bile acids analysis}

Fasting Serum samples were taken from women and measured twice every week since the gestational age of 32 weeks until caesarean section. Total bile acids were assessed by using an enzymatic method (Kit No Bl 3863, Randox Laboratoires Ltd, United Kingdom) on a Hitachi 7180 biochemistry analyzer (Hitachi, Tokyo, Japan).

\section{RNA preparation}

Freshly isolated placental tissues were divided and dissected separately on ice-cooled RNase-free surfaces. Each sample was placed into an individual tube before addition of $1 \mathrm{~mL}$ of TRIzol reagent (Invitrogen), followed by homogenization with a Polytron mixer (Kinematica). Total RNA was extracted according to manufacturer's instructions (Invitrogen). For DNA microarray, ten pregnant women in each group were randomly divided into two sub-groups and equal amounts of RNA from five individuals in each subgroup were pooled for one array to reduce the variations among different individuals $[14,15]$.

\section{DNA microarray and hybridization}

Agilent Human $4 \times 44 \mathrm{~K}$ Gene Expression Arrays, which target 27958 Entrez Gene RNAs, were employed in this study. Sequences were compiled from a broad survey of sources and were subsequently verified and optimized by alignment to the assembled human genome. Coupled with Agilent's probe selection and robust validation processes, this design delivers increased data quality and less redundancy in gene coverage.

Sample labeling and array hybridization were performed according to the Agilent One-Color Microarray- 


\begin{tabular}{lccc}
\hline A & & & \\
\hline Group & Healthy & Mild ICP & Severe ICP \\
\hline Maternal age (year) & $27.5 \pm 3.2$ & $26.8 \pm 3.7$ & $28.6 \pm 4.2$ \\
Gestational age (week) & $39.2 \pm 0.9$ & $37.8 \pm 1.2$ & $36.6 \pm 1.3$ \\
Birth weight (g) & $3350 \pm 139$ & $3185 \pm 128$ & $3075 \pm 105$ \\
Total bile acids $(\mu \mathrm{M})$ & $3.4 \pm 0.4$ & $27.2 \pm 2.6$ & $61.1 \pm 3.1$ \\
\hline
\end{tabular}

\section{B}

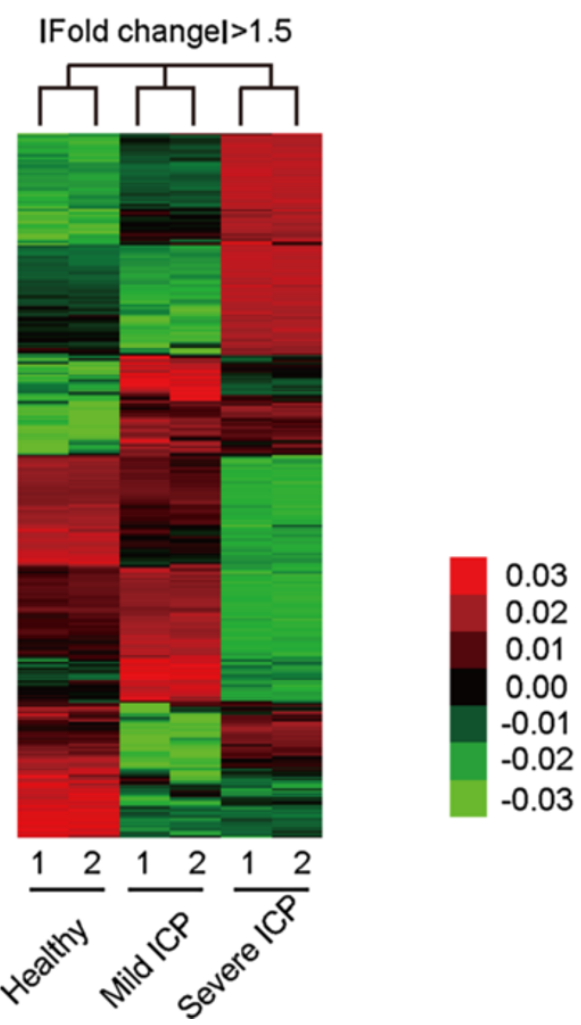

Figure 1 Hierarchical clustering of differentially expressed genes in placentas from healthy pregnancies and women with ICP. (A) Clinical features of healthy women and women with mild or severe ICP. 10 pregnant women were recruited for each group, and data are shown as mean \pm s.d. $(n=10)$. (B) Hierarchical clustering of genes whose expression was altered by more than 1.5 -fold in ICP placentas comparing to those in healthy placentas. Red and green represent individual genes that were differentially up-regulated or down-regulated, respectively.

Based Gene Expression Analysis protocol (Agilent Technology). Briefly, total RNA from each sample was linearly amplified and labeled with Cy3-UTP. The labeled cRNAs were purified using the RNeasy Mini Kit (QIAGEN). The concentration and specific activity of the labeled cRNAs (pmol Cy3/ $\mu \mathrm{g}$ cRNA) were measured using a NanoDrop ND-1000. One microgram of each labeled cRNA was fragmented by addition of $11 \mu \mathrm{l} 10 \times$ Blocking Agent and $2.2 \mu \mathrm{l} 25 \times$ Fragmentation Buffer followed by heating at $60^{\circ} \mathrm{C}$ for $30 \mathrm{~min}$. Finally, $55 \mu \mathrm{l} 2 \times$ GE Hybridization buffer was added to dilute the labeled cRNA. One hundred microliters of hybridization solution was dispensed into the gasket slide, which was assembled with the gene expression microarray slide, followed by incubation for 17 hours at $65^{\circ} \mathrm{C}$ in an Agilent Hybridization Oven. The hybridized arrays were washed, fixed, and scanned with the Agilent DNA Microarray Scanner (part number G2505B). 
Data analysis and differentially expressed genes sorting Agilent Feature Extraction software (version 11.0.1.1) was used to analyze acquired array images. Quantile normalization and subsequent data processing were performed using the GeneSpring GX v12.0 software package. Genes that had flags in at least two out of six arrays were chosen for further analysis. Detailed gene expression data are available at the GEO website under accession number GSE46157.

To sort the differentially expressed genes in mild ICP and severe ICP compared with healthy pregnancy, microarray data were analyzed by using linear models and empirical Bayes method, which is considered to be able to generate a reliable statistical inference when the number of arrays is small $[16,17]$. This method is similar to a standard $t$ test for each probe except that the SES are moderated across genes to get more stable results, which prevents a gene with a very small fold change from being judged as differentially expressed due to an accidentally small residual SD. The resulting $p$ values were further adjusted using the BH FDR algorithm [18]. Subsequently, differentially expressed genes were sorted according to fold change $( \pm 1.5$-fold or greater), followed by secondary selection based on $p$-value and FDR threshold ( $\mathrm{p}<0.05, \mathrm{FDR}<0.05$, controlling the expected FDR to no more than $5 \%$ ).

\section{Hierarchical clustering of differentially expressed genes}

Hierarchical clustering was performed using the Agilent GeneSpring GX software (version 12.0), and the results were expressed as a dendrogram in which genes and samples with a similar expression pattern formed clusters.

\section{Gene Ontology (GO) analysis}

Gene Ontology (GO) analysis was applied to characterize the primary functions of the differentially expressed genes based on the key functional classification of NCBI [19]. Fisher's exact test was used to classify the GO category. A $p$-value was calculated for each GO term for all differentially expressed genes and subsequently corrected by falsediscovery rate (FDR) [20]. A smaller FDR is associated with a smaller error in judging the $p$-value. Next, enrichment analysis was performed to find GO terms with more specific functions. The enrichment $R_{e}$ was given by $R_{e}=$ $\left(n_{f} / n\right) /\left(N_{f} / N\right)$, where $n_{f}$ is the number of differential genes within the particular category, $n$ is the total number of genes within the same category, $N_{f}$ is the number of differentially expressed genes in the entire microarray, and $N$ is the total number of genes on the microarray.

\section{Gene co-expression network}

To identify modules of co-expressed genes [21], we built the gene co-expression network according to the normalized signal intensities of specific genes. We calculated the Pearson's correlation for each pair of genes and chose those whose values fit the selection criterion to construct the network [22]. Degree of centrality, defined as the number of links between one node and the others, was used as the simplest and most important measure of the centrality of a gene within a network, which in turn determines that gene's relative importance. In addition, the kcore (from graph theory) was introduced to simplify the graph-topology analysis for the purposes of locating the core regulatory factors (genes) in the gene co-expression network. The k-core of a gene co-expression network usually contains a cohesive group of genes [23-25]. A k-core sub-network with a higher $\mathrm{k}$-core level is considered to have core status within a large-scale gene network.

\section{Quantitative real-time PCR (qPCR)}

Total RNA of each placental sample was prepared as described in RNA preparation. Synthesis of cDNA was carried out using Reverse Transcriptase M-MLV (RNase H-; TaKaRa) and oligo-dT as the primer. QPCR was performed on an Applied Biosystems 7500 Fast Real-Time PCR System using Power SYBR Green PCR Master Mix (Applied Biosystems, Foster City, CA, USA). Primer pairs used were listed in Additional file 1: Table S1. Reactions were performed in triplicate under standard thermocycling conditions (one cycle of $94^{\circ} \mathrm{C}$ for $4 \mathrm{~min}$, followed by 45 cycles of $94^{\circ} \mathrm{C}$ for $30 \mathrm{~s}, 58^{\circ} \mathrm{C}$ for $30 \mathrm{~s}$, and $72^{\circ} \mathrm{C}$ for $40 \mathrm{~s}$ ), and the mean threshold cycle number was used.

\section{Histology and immunohistochemistry}

Freshly isolated placental tissues were fixed with 4\% PFA overnight at $4^{\circ} \mathrm{C}$, embedded in paraffin, sectioned at $4-\mu \mathrm{m}$ thickness, and followed by hematoxylin and eosin staining.

Immunohistochemical analyses were performed using the LSAB kit (ZSGB-Bio, Beijing, China). Briefly, paraffin sections were deparaffinized in xylene and rehydrated sequentially in ethanol. The sections were microwaved in EDTA buffer ( $\mathrm{pH}$ 8.0) to retrieve antigens, incubated in $3 \% \mathrm{H}_{2} \mathrm{O}_{2}$ (10 min, room temperature) to inactivate endogenous peroxidase. Sections were incubated with blocking solution $\left(15 \mathrm{~min}, 37^{\circ} \mathrm{C}\right)$, followed by incubation in mouse anti-human CD45 antibody (1:200, 304002, Biolegend) diluted in blocking buffer (overnight, $4^{\circ} \mathrm{C}$ ). The samples were then incubated with biotinylated secondary antibody $\left(15 \mathrm{~min}, 37^{\circ} \mathrm{C}\right)$, followed by incubation with streptavidin conjugated with $\operatorname{HRP}\left(15 \mathrm{~min}, 37^{\circ} \mathrm{C}\right)$ and staining with DAB substrate. Nuclei were lightly counterstained with hematoxylin. Finally, slides were dehydrated sequentially in ethanol, cleared with xylenes, and mounted with neutral resin.

For immunofluorescence staining, tissue was fixed in $4 \%$ PFA overnight at $4^{\circ} \mathrm{C}$, immersed in sucrose solutions, embedded in OCT (Tissue Tek) and sectioned. Frozen sections were incubated with 10\% FBS (PBS) to block nonspecific binding sites (1 h, RT) and further incubated 
with mouse anti-humam CD19 antibody (Alexa Fluor ${ }^{\circ}$ 700 conjugated, HIB19, 1:200, BD Pharmingen) or mouse anti-human CD3 antibody (FITC conjugated, APA1/1, 1:200, BD Pharmingen) at $4^{\circ} \mathrm{C}$ overnight. Nuclei were stained with DAPI.

The stained sections were imaged using an Olympus BX51 microscope coupled with an Orca CCD camera (Q-IMAGING).

\section{Quantifications of blood vessels and immune cells}

The HE staining image was used for blood vessel quantification. Ten sections of the terminal villi at the same position in each placenta were examined and 5 random fields in each slide were selected (50 fields total for each placenta) to avoid areas of placental infarction and intervillous fibrin deposition, arterial vessels forming the primary stem and anchoring villi; and histological artifact. The selected fields were then analyzed using ImageJ to count the number of capillaries in each villus. Only terminal villi showing outlines completely within the microscopic field were analyzed. The number of capillaries per villus was calculated by the total number of capillaries divided by the number of terminal villi in 50 fields for each placental sample.

For immune cell quantification, ten sections of the terminal villi at the same position in each placenta were examined and the number of different immune cells in 5 random fields in each section were counted (50 fields

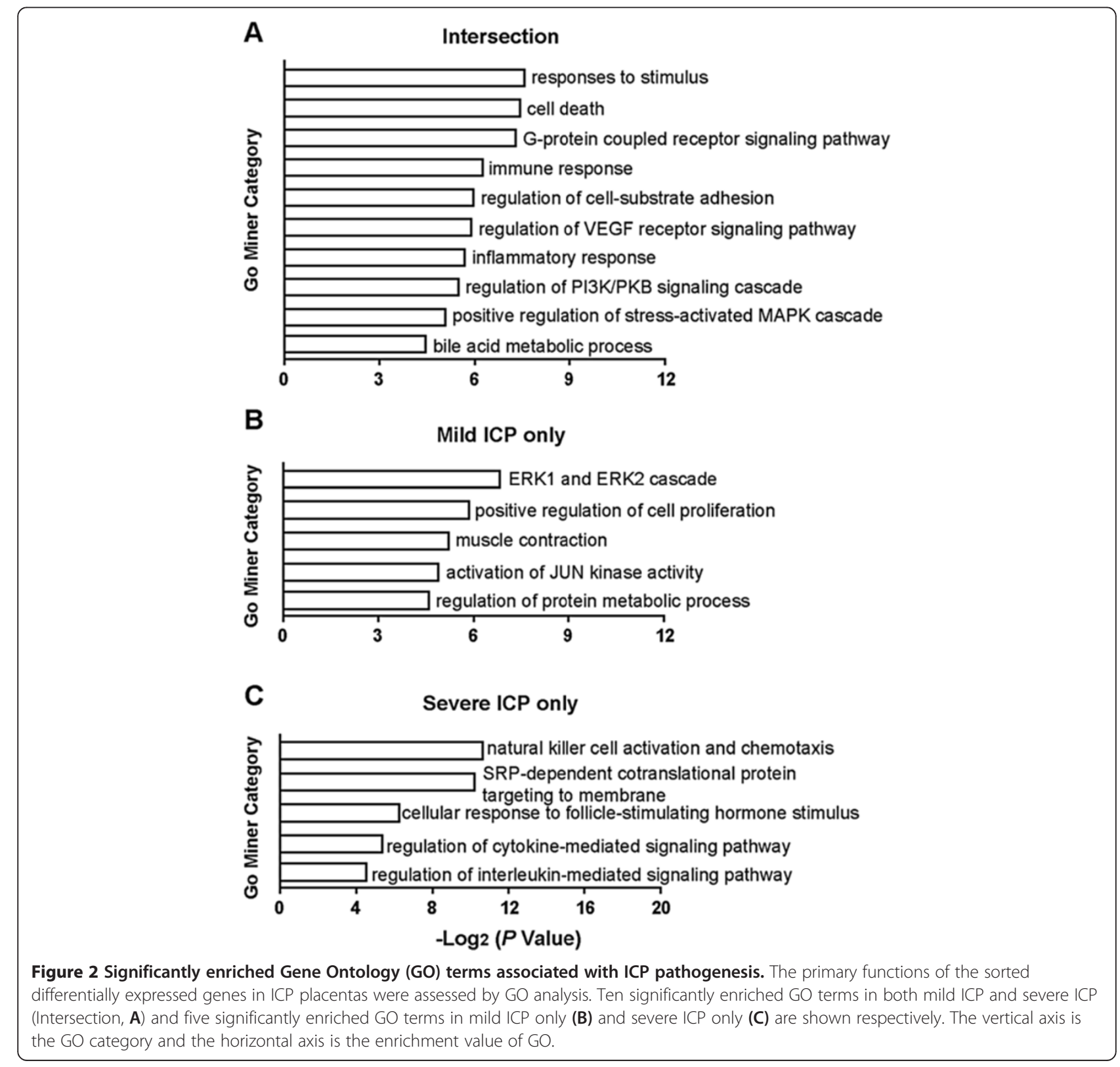


total for each placenta). The average of cell number per field was calculated for each placenta.

\section{Statistical analysis}

$P$ values of qPCR results, immune cell quantification and blood vessel quantification were calculated by analysis of variance (ANOVA) with Dunnett post-tests. Significant differences are indicated as: *, $p<0.05$; **, $p<0.01$. Each experiment was repeated independently for three times.

\section{Results and discussion}

First we employed DNA microarray to identify differentially expressed genes in mild ICP and severe ICP compared to those in healthy pregnancies. Hierarchical clustering of genes whose expression was altered by more than 1.5 -fold revealed that gene expression profiles of the two parallel microarrays in each group were highly consistent, indicating the reproducibility of microarray data (Figure 1B).
Moreover, as can be observed from the heat map of clustered genes, the three groups of pregnancies had distinctive gene-expression profiles (Figure 1B).

Subsequently, we applied Gene Ontology (GO) analysis to assess the primary functions of the differentially expressed genes [19] (Figure 2A-C). There was extensive overlap between the GO terms of mild ICP and severe $\mathrm{ICP}$, indicating a strong correlation between mild ICP and severe ICP (Figure 2A). In addition, it is worthwhile to note that the GO terms specifically enriched in severe ICP were mainly related to immune-related signaling pathways (Figure $2 \mathrm{C}$ ).

To further characterize the core regulatory genes involved in the pathogenesis of ICP, genes associated with the significantly enriched GO terms were used to construct gene-interaction and gene co-expression networks $[21,26]$ (Figure 3 and Additional file 2: Figure S1). Detailed genegene relationships with the degree of each gene from gene-

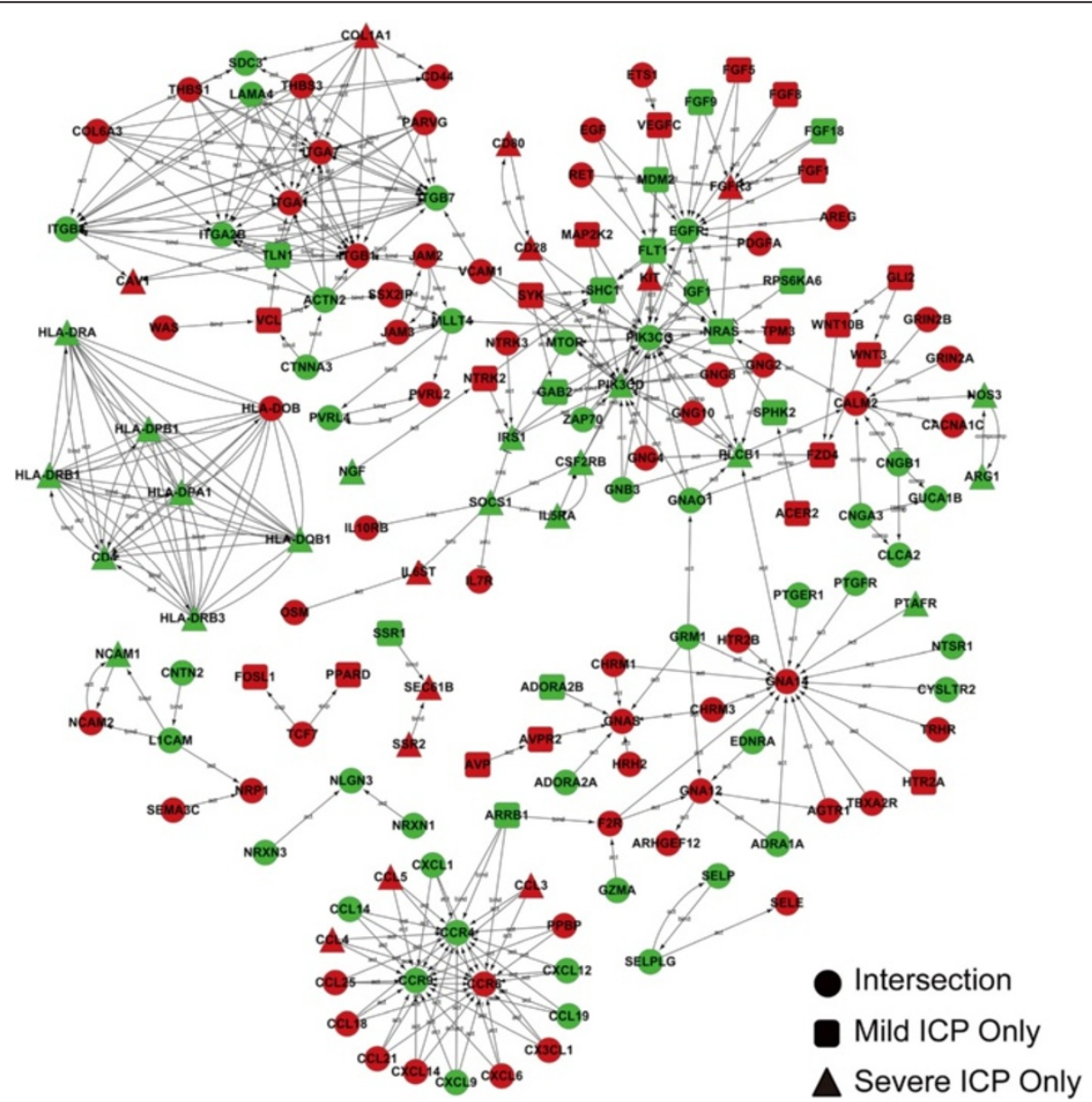

Figure 3 Gene-interaction network. Genes associated with the significantly enriched GO terms were analyzed by gene-interaction network, which was built according to the relationships among genes, proteins, and compounds in the KEGG database. Circles, squares, and triangles represent, respectively, genes differentially regulated in both mild ICP and severe ICP (Intersection), mild ICP only, and severe ICP only. Red and green represent genes that were differentially up-regulated or down-regulated, respectively. Detailed gene-gene relationships with the degree of each gene are shown in Additional file 3: Spreadsheet S1. 
interaction network and gene co-expression network are shown in Additional file 3: Spreadsheets S1 and Additional file 4: Spreadsheets S2, respectively. These two networks identified the following 19 core regulatory genes: CCL3, CCL25, CXCL6, CXCL14, CCR4, CCR6, CCR9 and IL-7R involved in immune response; VEGFC, FZD4, FGF9, FGF18, $I T G B 3$, and FLT1 involved in VEGF signaling pathway; and GNAS, GNA12, GNA14, EGF, and EGFR involved in Gprotein-coupled receptor (GPCR) signaling.

Next we used qPCR to confirm the expression patterns of the identified 19 core regulatory genes in 30 individuals in all three groups (Additional file 1: Table S1). 14 of 19 detected genes exhibited expression patterns consistent with those from the microarray data, confirming the accuracy of the microarray data (Figure 4).

Despite decades of research, pregnancy is characterized by quite an unusual number of rather frequent conditions with unknown etiology. Although previous studies have provided some information on the altered gene expression profile in ICP placentas [14,27], they did not discriminate between patients with mild ICP and severe ICP. In this study, to further reveal the influence of different bile acid concentration in the pathogenesis of ICP, we sub-grouped the ICP patients into mild and severe ICP according to their serum bile acid level. In addition, as ICP women delivered at earlier gestational stage than healthy pregnant women (Figure 1A), it is possible that different gestational ages of healthy and ICP groups might affect the mRNA expression of placentas. Nevertheless, we were not able to collect placentas from healthy women and women with ICP of the same gestational age in clinical cases, which is a common problem encountered by other studies on the placental gene expression profiles of healthy pregnant women and women with ICP [14,27].

Like Gleicher has pointed out in a number of publications, these unexplained conditions of pregnancy exhibit characteristics similar to immunological conditions observed in organ transplant rejection [28] and/or graft versus host disease (GVHD) [29], therefore ICP may represent the complications of normal tolerance of the fetal semiallograft by the maternal immune system [30]. Herein, our microarray results revealed that genes associated with immune response, CXCL6, CXCL14 and IL-7R were upregulated in mild ICP and further up-regulated in severe
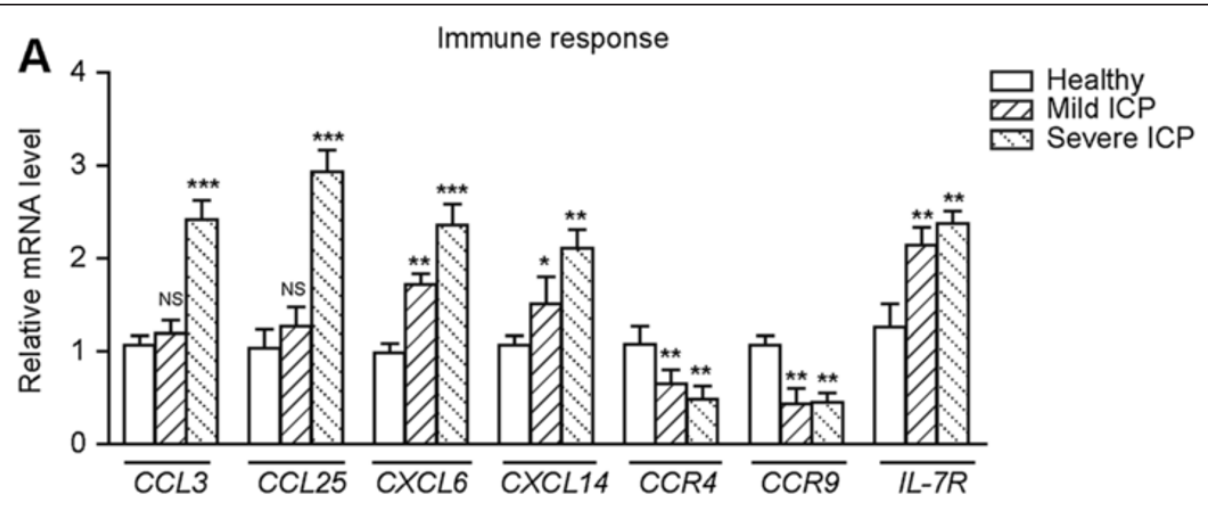

B

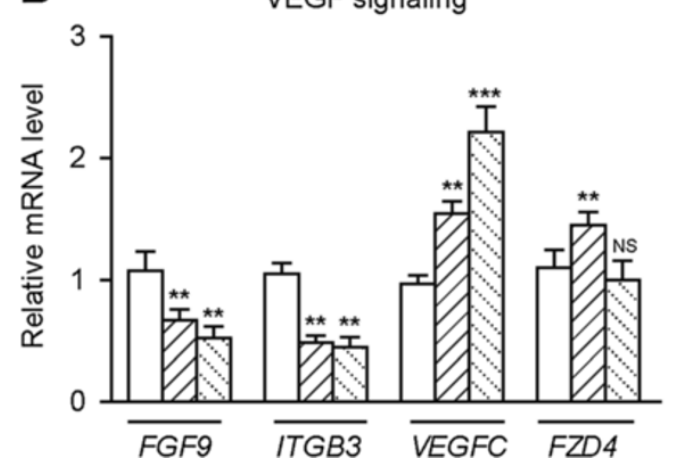

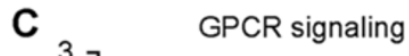

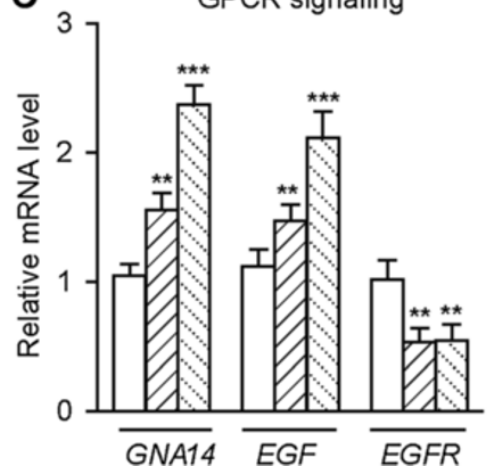

Figure 4 Validation of the identified core regulatory genes associated with ICP pathogenesis by qPCR. Genes with expression patterns in placental tissues from healthy group, mild ICP, and severe ICP (10 pregnancies per group) consistent with those in the microarray data are shown: Genes involved in (A) immune response, (B) VEGF signaling and (C) G-protein-coupled receptor (GPCR) signaling. Relative expression of each target gene was normalized to GAPDH, and expression levels in the healthy group were defined as 1 . All reactions were performed in triplicate, and data are presented as mean \pm s.d. $(n=10) .{ }^{*} p<0.05 ;{ }^{* *} p<0.01 ;{ }^{* *} p<0.001 ; \mathrm{NS}$, not significant. Primer pairs used were listed in Additional file 1: Table S1. 
ICP, while CCL3 and CCL25 were only up-regulated in severe ICP, which suggest that abnormal immune response in patients' placentas might be positively correlated to the severity of ICP. To explore this implication, we compared the infiltration of CD45 positive leukocytes in placentas from healthy pregnant women and patient with mild or severe ICP. Few leukocytes were present in normal placentas, whereas placentas from mild ICP exhibited more leukocytes aggregation, and placentas from severe ICP displayed massive leukocytes infiltration (Figure 5A). These results are consistent with the gradually elevated expression levels of immune-related genes in mild and severe ICP placentas, and suggest that ICP is associated with immune activation. To further determine which types of immune cells were recruited into placenta in ICP, we stained CD3 positive T cells and CD19 positive B cells in placentas. Compared with normal placentas, placentas from mild ICP showed significantly increased infiltration of both T cells and B cells, and the $\mathrm{T} / \mathrm{B}$ cells infiltration was further augmented in placenta from patient with severe ICP (Figure 5B). Taken together, our study suggests that ICP is an immunological/inflammatory condition and provides an evidence to support such an interpretation that ICP might be either an allograft rejection mechanism or, more likely, a counterpart to GVHD in organ transplantation.

Development of a normally functioning placental vascular network to support increasing oxygen and metabolic demands of the growing fetus is necessary for a successful pregnancy. This requires substantial coordination among different vascular endothelial cell-specific growth factors and cell types and is exquisitely dependent on signals exchanged between these cells [31]. As revealed by this study, ICP was accompanied by aggregated immune cell infiltration and gene expression levels of FGF9 and ITGB3 were significantly down-regulated in ICP placentas (Figure 5A and Figure 4B). Given the fact that immune cells are able to intimately regulate vessel formation and function [32] and considering the essential role of FGF9 and integrin $\beta 3$ in
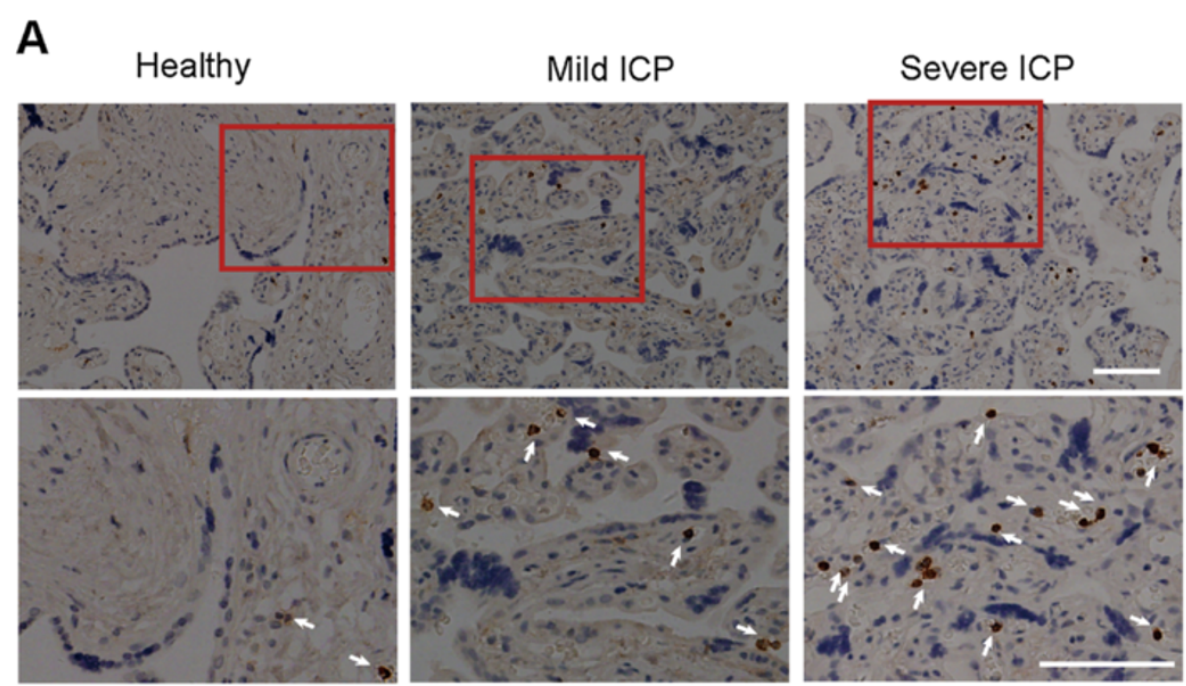

B
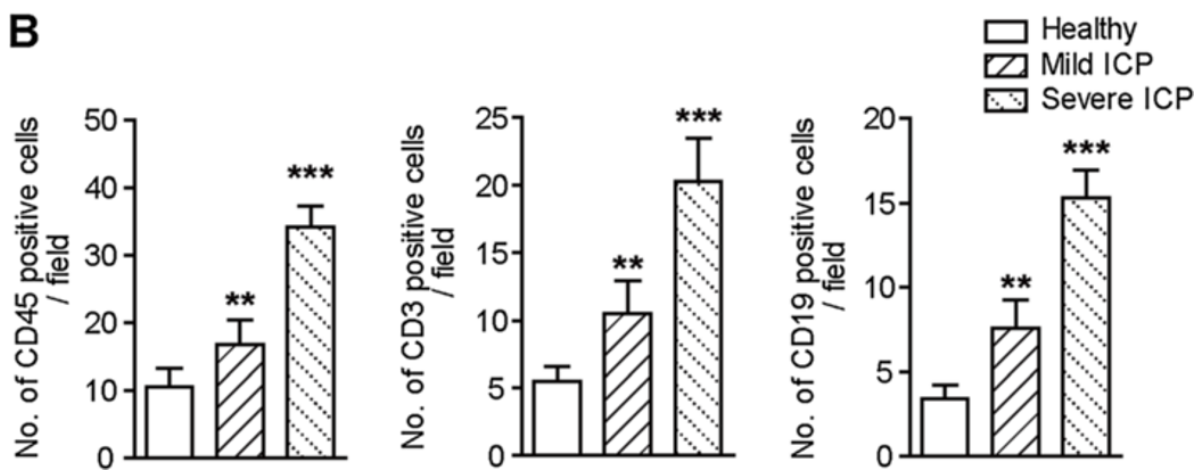

Figure 5 Immune cell infiltration in placental tissues from healthy group, mild ICP, and severe ICP. (A) Representative images of immunohistochemistry staining for the leukocyte marker CD45 in placental tissues from healthy group, mild ICP, and severe ICP (10 pregnancies per group). Brown staining (arrows) indicates reactivity. Scale bars represent $100 \mu \mathrm{m}$. (B) Quantification of leukocytes (CD45 positive), T cell (CD3 positive) and B cell (CD19 positive) in placental tissues from healthy group, mild ICP, and severe ICP (10 pregnancies per group). Data are presented as mean \pm s.d. $(n=10) .{ }^{* *} p<0.01 ;{ }^{* * *} p<0.001$. 


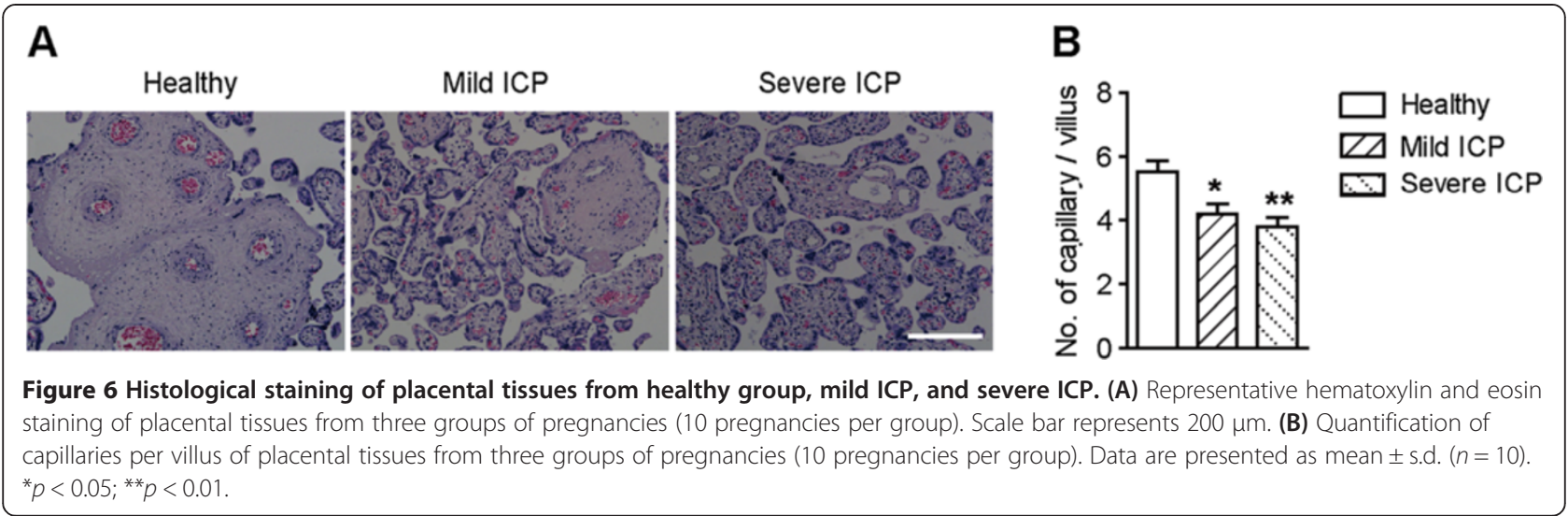

vasculogenesis [33] and angiogenesis [34], we postulated that formation and maturation of placental blood vessels might be impaired under conditions of ICP. To test this hypothesis, we subjected placental tissues from healthy and ICP pregnancies to hematoxylin and eosin staining to visualize the vascular structure. Normal placentas exhibited well-organized vascular structure consisting of large vessels (Figure 6A). By contrast, placentas from mild or severe ICP exhibited fewer and smaller blood vessels in each villus (Figure 6A,B), indicating disturbed placental vascular formation and deficient vascular maturation. Interestingly, placental gene expression of $F Z D 4$, which plays an essential role in vascular development [35], was increased in mild ICP but not in severe ICP (Figure 4B). Since previous studies have shown that FZD4 expression can be induced by hypoxia [35], it is speculative that up-regulation of FZD4 in mild ICP may serve as a mechanism to compensate for fluctuations of oxygen and hypoxia that result from lack of vessel formation and blood supply. However, this type of compensation does not exist under condition of severe ICP, which may explain, albeit partially, observations of dilated maternal vascular lacunae in severe ICP placentas (Figure 6A). Moreover, it is worthwhile to note that, gene expression of VEGFC was up-regulated in both mild ICP and severe ICP (Figure 4B). Given the function of VEGFC in blood vessel formation [36,37] and the fact that high levels of bile acids exert a constrictive effect on isolated human placental chorionic veins [38], it is tempting to speculate that VEGFC expression was up-regulated as a compensation for hypoxia induced by elevated concentrations of bile acids in ICP placentas.

Additionally, in ICP placentas, we observed differential regulation of critical regulatory genes involved in GPCR signaling, including EGF, EGFR, and genes encoding Gprotein subunits such as GNA14 (Figure 4C). Because GPCR signaling plays important roles in immune response, this finding implies a potential link between GPCR signaling and the abnormal immune activation in placentas of ICP patients.

\section{Conclusions}

In conclusion, our study provides a system-level insight into the placental gene-expression profiles of women with mild or severe ICP, and reveals multiple molecular pathways in immune response and blood vessel formation that might contribute to ICP pathogenesis. These findings should facilitate elucidation of the mechanisms of ICP development and may suggest therapeutic strategies for management of this disease.

\section{Additional files}

Additional file 1: Table S1. Primer pairs used for quantitative real-time
PCR.
Additional file 2: Figure S1. Gene co-expression network. Genes
associated with the significantly enriched GO terms were analyzed by
gene co-expression network using k-core algorithm. Node size
represents the strengths of relationships among nodes, and edges
between two nodes represent interactions between genes. Genes that
connect more edges (i.e., to more genes) play more central roles within
the network. Circles, squares, and triangles represent, respectively, genes
differentially regulated in both mild ICP and severe ICP (Intersection),
mild ICP only, and severe ICP only. Red and green represent genes that
were differentially up-regulated or down-regulated, respectively.
Detailed gene-gene relationships with degree and k-core of each gene
are shown in Additional file 3: Spreadsheet S2.
Additional file 3: Spreadsheet S1. The detailed gene-gene
relationships in the gene-interaction network.
Additional file 4: Spreadsheet S2. The detailed gene-gene
relationships from the gene co-expression network.

\section{Abbreviations}

ICP: Intrahepatic cholestasis of pregnancy; HELLP: Elevated liver enzymes and low platelets; GO: Gene ontology; FDR: False-discovery rate; qPCR: Quantitative real-time PCR; ANOVA: Analysis of variance; GPCR: G-protein-coupled receptor; GVDH: Graft versus host disease.

\section{Competing interests}

There is no conflict of interest that could be perceived as prejudicing the impartiality of the research reported.

\section{Authors' contributions}

All authors contributed to the concept, design, acquisition of data or analysis, interpretation of data, drafting or revising the content and approved the final version. 


\section{Authors' information}

QiaoLing Du, M.D., Chief physician, Department of Obstetrics, Shanghai First Maternity and Infant Hospital, Tongji University School of Medicine, China. YouDong Pan, Ph.D., post-doc, State Key Laboratory of Cell Biology, Institute of Biochemistry and Cell Biology, Shanghai Institutes for Biological Sciences, Chinese Academy of Sciences, China.

YouHua Zhang, Ph.D. candidate, State Key Laboratory of Cell Biology, Institute of Biochemistry and Cell Biology, Shanghai Institutes for Biological Sciences, Chinese Academy of Sciences, China.

HaiLong Zhang, Ph.D. candidate, State Key Laboratory of Cell Biology, Institute of Biochemistry and Cell Biology, Shanghai Institutes for Biological Sciences, Chinese Academy of Sciences, China.

YaJuan Zheng, M.D., technician State Key Laboratory of Cell Biology, Institute of Biochemistry and Cell Biology, Shanghai Institutes for Biological Sciences, Chinese Academy of Sciences, China.

Ling Lu, Ph.D. candidate, State Key Laboratory of Cell Biology, Institute of Biochemistry and Cell Biology, Shanghai Institutes for Biological Sciences, Chinese Academy of Sciences, China.

JunLei Wang, Ph.D. candidate, State Key Laboratory of Cell Biology, Institute of Biochemistry and Cell Biology, Shanghai Institutes for Biological Sciences, Chinese Academy of Sciences, China.

Tao Duan, M.D., Professor, Director, Department of Obstetrics, Shanghai First Maternity and Infant Hospital, Tongji University School of Medicine, China. JianFeng Chen, Ph.D., Principle Investigator, Professor, State Key Laboratory of Cell Biology, Institute of Biochemistry and Cell Biology, Shanghai Institutes for Biological Sciences, Chinese Academy of Sciences, China.

\section{Acknowledgements}

The authors thank all the pregnant women who participated in this study and gratefully acknowledge the support of the SA-SIBS scholarship program.

\section{Funding}

This work was supported by grants: National Basic Research Program of China (2010CB529703, 2014CB541905), National Natural Science Foundation of China (31190061, 31271487, 81170628), Science and Technology Commission of Shanghai Municipality (11JC1414200, 12ZR1423700), and SKLCB (KF2012002).

Supplementary information is linked to the online version of the paper on BMC Medical Genomics website.

Received: 25 September 2013 Accepted: 3 July 2014

Published: 7 July 2014

\section{References}

1. Arrese M, Reyes H: Intrahepatic cholestasis of pregnancy: a past and present riddle. Ann Hepatol 2006, 5(3):202-205.

2. Arrese M, Macias Rl, Briz O, Perez MJ, Marin JJ: Molecular pathogenesis of intrahepatic cholestasis of pregnancy. Expert Rev Mol Med 2008, 10:e9.

3. Lausman AY, Al-Yaseen E, Sam D, Nitsch R, Barrett JF, Chan WS: Intrahepatic cholestasis of pregnancy in women with a multiple pregnancy: an analysis of risks and pregnancy outcomes. J Obstet Gynaecol Can 2008, 30(11):1008-1013.

4. Beuers U, Pusl T: Intrahepatic cholestasis of pregnancy-a heterogeneous group of pregnancy-related disorders? Hepatology 2006, 43(4):647-649.

5. Laatikainen T, Tulenheimo A: Maternal serum bile acid levels and fetal distress in cholestasis of pregnancy. Int J Gynaecol Obstet 1984, 22(2):91-94.

6. Glantz A, Marschall HU, Mattsson LA: Intrahepatic cholestasis of pregnancy: Relationships between bile acid levels and fetal complication rates. Hepatology 2004, 40(2):467-474.

7. Williamson C, Miragoli M, Sheikh Abdul Kadir S, Abu-Hayyeh S, Papacleovoulou G, Geenes V, Gorelik J: Bile acid signaling in fetal tissues: implications for intrahepatic cholestasis of pregnancy. Dig Dis 2011, 29(1):58-61.

8. Colombo C, Zuliani G, Ronchi M, Breidenstein J, Setchell KD: Biliary bile acid composition of the human fetus in early gestation. Pediatr Res 1987, 21(2):197-200.

9. Geenes VL, Lim YH, Bowman N, Tailor H, Dixon PH, Chambers J, Brown L, Wyatt-Ashmead J, Bhakoo K, Williamson C: A placental phenotype for intrahepatic cholestasis of pregnancy. Placenta 2011, 32(12):1026-1032.

10. Colombo C, Roda A, Roda E, Buscaglia M, Dell'Agnola CA, Filippetti P, Ronchi M, Sereni F: Correlation between fetal and maternal serum bile acid concentrations. Pediatr Res 1985, 19(2):227-231.
11. Laatikainen TJ: Fetal bile acid levels in pregnancies complicated by maternal intrahepatic cholestasis. Am J Obstet Gynecol 1975, 122(7):852-856.

12. Perez MJ, Macias RI, Marin JJ: Maternal cholestasis induces placental oxidative stress and apoptosis. Protective effect of ursodeoxycholic acid. Placenta 2006, 27(1):34-41.

13. Wikstrom Shemer E, Thorsell M, Ostlund E, Blomgren B, Marschall HU: Stereological assessment of placental morphology in intrahepatic cholestasis of pregnancy. Placenta 2012, 33(11):914-918.

14. Wei J, Wang $H$, Yang $X$, Dong M, Wang Z: Altered gene profile of placenta from women with intrahepatic cholestasis of pregnancy. Arch Gynecol Obstet 2010, 281(5):801-810.

15. Cristofalo VJ: A DNA chip off the aging block. Nat Med 2000, 6(5):507.

16. Smyth GK: Linear models and empirical bayes methods for assessing differential expression in microarray experiments. Stat Appl Genet Mol Biol 2004, 3:3.

17. Saeys $Y$, Inza I, Larranaga P: A review of feature selection techniques in bioinformatics. Bioinformatics 2007, 23(19):2507-2517.

18. Benjamini Y, Hochberg Y: Controlling the False Discovery Rate: A Practical and Powerful Approach to Multiple Testing. Journal of the Royal Statistical Society Series B Methodological 1995, 57(1):289-300.

19. Ashburner M, Ball CA, Blake JA, Botstein D, Butler H, Cherry JM, Davis AP, Dolinski K, Dwight SS, Eppig JT, Harris MA, Hill DP, Issel-Tarver L, Kasarskis A, Lewis S, Matese JC, Richardson JE, Ringwald M, Rubin GM: Gene ontology: tool for the unification of biology. The Gene Ontology Consortium. Nat Genet 2000, 25(1):25-29.

20. Dupuy D, Bertin N, Hidalgo CA, Venkatesan K, Tu D, Lee D, Rosenberg J, Svrzikapa N, Blanc A, Carnec A, Carvunis AR, Pulak R, Shingles J, Reece-Hoyes J, Hunt-Newbury R, Viveiros R, Mohler WA, Tasan M, Roth FP, Le Peuch C, Hope IA, Johnsen R, Moerman DG, Barabasi AL, Baillie D, Vidal M: Genome-scale analysis of in vivo spatiotemporal promoter activity in Caenorhabditis elegans. Nat Biotechnol 2007, 25(6):663-668.

21. Pujana MA, Han JD, Starita LM, Stevens KN, Tewari M, Ahn JS, Rennert G, Moreno V, Kirchhoff T, Gold B, Assmann V, Elshamy WM, Rual JF, Levine D, Rozek LS, Gelman RS, Gunsalus KC, Greenberg RA, Sobhian B, Bertin N, Venkatesan K, Ayivi-Guedehoussou N, Sole X, Hernandez P, Lazaro C, Nathanson KL, Weber BL, Cusick ME, Hill DE, Offit K, et al: Network modeling links breast cancer susceptibility and centrosome dysfunction. Nat Genet 2007, 39(11):1338-1349.

22. Prieto C, Risueno A, Fontanillo C, de las Rivas J: Human gene coexpression landscape: confident network derived from tissue transcriptomic profiles. PLoS One 2008, 3(12):e3911.

23. Barabasi AL, Oltvai ZN: Network biology: understanding the cell's functional organization. Nat Rev Genet 2004, 5(2):101-113.

24. Ravasz E, Somera AL, Mongru DA, Oltvai ZN, Barabasi AL: Hierarchical organization of modularity in metabolic networks. Science 2002, 297(5586):1551-1555.

25. Carlson MR, Zhang B, Fang Z, Mischel PS, Horvath S, Nelson SF: Gene connectivity, function, and sequence conservation: predictions from modular yeast co-expression networks. BMC Genomics 2006, 7:40.

26. Spirin V, Mirny LA: Protein complexes and functional modules in molecular networks. Proc Natl Acad Sci USA 2003, 100(21):12123-12128.

27. Floreani A, Caroli D, Lazzari R, Memmo A, Vidali E, Colavito D, D'Arrigo A, Leon A, Romero R, Gervasi M: Intrahepatic Cholestasis Of Pregnancy: New Insights Into Its Pathogenesis. J Matern Fetal Neonatal Med 2013, 26:1410-1415.

28. Gleicher N, Elkayam U: Peripartum cardiomyopathy, an autoimmune manifestation of allograft rejection? Autoimmun Rev 2009, 8(5):384-387.

29. Gleicher N: Why much of the pathophysiology of preeclampsiaeclampsia must be of an autoimmune nature. Am J Obstet Gynecol 2007, 196(1):5 e1-7.

30. Gleicher N, Barad DH: Gestational dermatosis shortly after implantation associated with parental class II HLA compatibility and maternal immune activation: preliminary report of a prospective case series. Dermatology 2011, 222(3):206-211.

31. Geva E, Ginzinger DG, Zaloudek CJ, Moore DH, Byrne A, Jaffe RB: Human placental vascular development: vasculogenic and angiogenic (branching and nonbranching) transformation is regulated by vascular endothelial growth factor-A, angiopoietin-1, and angiopoietin-2. J Clin Endocrinol Metab 2002, 87(9):4213-4224.

32. Noonan DM, De Lerma BA, Vannini N, Mortara L, Albini A: Inflammation, inflammatory cells and angiogenesis: decisions and indecisions. Cancer Metastasis Rev 2008, 27(1):31-40. 
33. White $\mathrm{AC}$, Lavine $\mathrm{K}$, Ornitz DM: FGF9 and $\mathrm{SHH}$ regulate mesenchymal Vegfa expression and development of the pulmonary capillary network. Development 2007, 134(20):3743-3752.

34. Hayashi H, Sano H, Seo S, Kume T: The Foxc2 transcription factor regulates angiogenesis via induction of integrin beta3 expression. $J$ Biol Chem 2008, 283(35):23791-23800.

35. Luhmann UF, Neidhardt J, Kloeckener-Gruissem B, Schafer NF, Glaus E, Feil S, Berger W: Vascular changes in the cerebellum of Norrin/Ndph knockout mice correlate with high expression of Norrin and Frizzled-4. Eur J Neurosci 2008, 27(10):2619-2628.

36. Cao Y, Linden P, Farnebo J, Cao R, Eriksson A, Kumar V, Qi JH, Claesson-Welsh L, Alitalo $K$ : Vascular endothelial growth factor $C$ induces angiogenesis in vivo. Proc Natl Acad Sci U S A 1998, 95(24):14389-14394.

37. Karkkainen MJ, Petrova TV: Vascular endothelial growth factor receptors in the regulation of angiogenesis and lymphangiogenesis. Oncogene 2000, 19(49):5598-5605.

38. Sepúlveda WH GC, Cruz MA, Rudolph MI: Vasoconstrictive effect of bile acids on isolated human placental chorionic veins. Eur J Obstet Gynecol Reprod Biol 1991, 42(3):5.

doi:10.1186/1755-8794-7-42

Cite this article as: Du et al.: Placental gene-expression profiles of intrahepatic cholestasis of pregnancy reveal involvement of multiple molecular pathways in blood vessel formation and inflammation. BMC Medical Genomics 2014 7:42.

\section{Submit your next manuscript to BioMed Central and take full advantage of:}

- Convenient online submission

- Thorough peer review

- No space constraints or color figure charges

- Immediate publication on acceptance

- Inclusion in PubMed, CAS, Scopus and Google Scholar

- Research which is freely available for redistribution 